\title{
Radio, Internet und Satellit zur Bekämpfung der Buschfeuer im Senegal
}

\section{Martin Faye}

\section{OpenEdition}

\section{Journals}

Electronic version

URL: http://journals.openedition.org/sjep/539

DOI: $10.4000 /$ sjep.539

ISSN: 1663-9677

\section{Publisher}

Institut de hautes études internationales et du développement

\section{Printed version}

Date of publication: 1 novembre 2003

Number of pages: 93-95

ISSN: 1660-5926

\section{Electronic reference}

Martin Faye, «Radio, Internet und Satellit zur Bekämpfung der Buschfeuer im Senegal »,

Schweizerisches Jahrbuch für Entwicklungspolitik [Online], 22-2 | 2003, Online erschienen am: 10 Juni

2010, abgerufen am 08 September 2020. URL : http://journals.openedition.org/sjep/539 ; DOI :

https://doi.org/10.4000/sjep.539

This text was automatically generated on 8 septembre 2020 .

(c) The Graduate Institute 


\title{
Radio, Internet und Satellit zur Bekämpfung der Buschfeuer im Senegal
}

\author{
Martin Faye
}

1 In Afrika werden schon seit Jahren Informations- und Kommunikationstechnologien (IKT) in einigen Projekten spontan eingesetzt. Ob es sich um eine neue, eine analoge, eine alte oder eine traditionelle Technologie handelt, ist nicht wichtig. Wichtig ist nur, dass die angestrebten Ziele effizient, nachhaltig und autonom erreicht werden. Dort wo leistungsfähige Computer verfügbar sind, begriffen die Bauernvereinigungen schnell, dass der Kauf eines Modems ihnen hilft, sich mit anderen Vereinigungen in anderen Regionen zu vernetzen. Sie benutzen, wie viele andere, die IKT, ohne es zu wissen.

In den armen Ländern werden die IKT natürlich in den Medien am meisten eingesetzt. In den Printmedien hat der PC die alten und komplizierten Drucktechnologien ersetzt. Beim Radio sind die alten Einrichtungen praktisch verschwunden. Da Tonbänder heute keine Massenware mehr sind, sind sie für kleine Radiostationen unerschwinglich geworden. Digitale Einrichtungen im Audiobereich dagegen sind billig, wenn sie auch weniger lang halten und eine Ausbildung für deren Unterhalt erforderlich ist. Kurz, der ganze Radiosektor-die regionalen wie die nationalen Sender-erlebt einen tief greifenden technologischen Wandel.

3 PC und Modem haben in den ländlichen Radiostudios Einzug gehalten. Sie werden unterschiedlich und in oft überraschender Weise eingesetzt: Die mündlichen Überlieferungen Afrikas werden gesammelt und auf stabilen Tonträgern (CD, DVD, Festplatte) gespeichert. Über die ländlichen Radiostationen wird Analphabeten die elektronische Post zugänglich, so dass die Briefpost in den abgelegenen Regionen beinahe überflüssig geworden ist. Das gilt auch für das Projekt „Die Buschfeuer in Westafrika besser in den Griff bekommen“. Das von der Direktion für Entwicklung und Zusammenarbeit (DEZA) unterstützte Projekt verbindet die lokalen Gemeinschaften, die technischen Dienste sowie das Centre de suivi écologique ( $\operatorname{CSE}^{1}$, Zentrum für 
ökologische Überwachung) in Dakar, eine senegalische Institution, die auf Satellitenüberwachung spezialisiert ist.

4 Es gibt wohl kaum eine „ältere Technologie“ als das prometheische Feuer! Die Bauern setzen diesen „Bulldozer der Armen“ ein, um zu roden, die Felder vorzubereiten, die Schlangen aus den Häusern zu vertreiben, aber auch für die Jagd und für WahrsageRiten. Das weitflächig entfachte Feuer ist auch der einzige wirkliche Beitrag Afrikas an die globale Erwärmung. Die Praxis der frühzeitigen Feuer, die von den meisten technischen Diensten in Westafrika befürwortet werden, ist aber akzeptabel und entspricht den vorkolonialen Traditionen. Sie werden angezündet, wenn das Gras noch feucht ist. Das erzeugt wenig Hitze, und die Natur kann sich ab dem ersten Regen der neuen Saison wieder erholen. Die späten Feuer dagegen, die bei trockenem Gras gelegt werden, zerstören Pflanzen und Böden.

Die Sendungen der ländlichen Radiostationen Afrikas sind sehr wirksam, denn sie haben sich der Bewahrung der mündlichen Überlieferung verschrieben. Seit langem machen sie die lokalen Gemeinschaften auf die verhängnisvollen Folgen der Buschbrände aufmerksam-leider mit wenig Erfolg. Die Sendungen können sogar kontraproduktive Auswirkungen haben, wenn mit den Feuern politische Unzufriedenheit ausgedrückt werden soll. Ein Diktator, welcher sie verboten hatte, wurde auf seiner Reise durchs Land überall von Bränden begleitet, die eindeutig keine Freudenfeuer waren.

$6 \quad$ Nun aber verhelfen die vernetzte Arbeit über das Internet und die Satellitenbilder zu mehr Effizienz. Namentlich dank der Hilfe der Europäischen Union kennt man die Satellitenüberwachung der Brände in Westafrika jetzt seit zehn Jahren. Zu bestimmten Jahreszeiten entsteht aufgrund der Satellitenbilder der Eindruck, ganz Afrika brenne. Diese Daten kamen aber bisher nicht bis zu den Basisgemeinschaften, und die technischen Dienste nutzten sie nicht. Deshalb wendet das Projekt die Praxis des partizipativen Radios an: Zuerst wird das lokale Wissen gesammelt, und wenn man es verstanden hat, wird es mit dem Wissen der „Experten“ verbunden.

7 Am Anfang ging es darum, die Landbevölkerung anzuhören-mit langen und zahlreichen Interviews mit offenen wie gezielten Fragen und mit Reportagen in den Dörfern. Dieses Tonmaterial wurde mit den Partnern im Feld (technische Dienste, NRO usw.) analysiert, um sicher zu sein, dass man den Standpunkt der Bevölkerung wirklich verstanden hatte. Die Leiter hatten Instruktionen erhalten, den Experten nicht allzu schnell Recht zu geben, sondern die Probleme der Landbevölkerung aufzuzeigen und Lösungen vorzuschlagen. Wenn zum Beispiel rituelle Feuer in der Trockenperiode wirklich nötig sind, können diese durchgeführt werden, aber nur mit den nötigen Schutzvorrichtungen.

8 Die Radiokampagne selber ist gezielter, lässt aber gleichzeitig die Bauern, Hirten und Jäger zu Wort kommen. Weisungen wie: „Wenn etwas zu verbrennen ist, muss das jetzt geschehen“, „Die Saison für die frühen Feuer ist vorbei, es darf kein Feuer mehr gemacht werden" bauen auf klaren, in Interviews gemachten Äusserungen auf, denn die besten Fachleute sind Bauern, die sich an andere Bauern wenden.

9 Das Aufspüren von Feuern aus der Ferne spielt vor, während und nach der Kampagne eine zentrale Rolle. Dank einer Brandkarte konnten die Radiosender bestimmt werden, die für die Kampagne geeignet sind. Schliesslich machten ländliche Stationen in sieben Staaten Westafrikas (Benin, Burkina Faso, Guinea, Gambia, Mali, Niger und Senegal) mit. Die monatlich herausgegebenen Brandkarten wurden den Gemeinschaften über E- 
Mail und ländliche Radiostationen mitgeteilt. So spürt die Landbevölkerung, dass sie von oben beobachtet wird, was in ihren Augen ein Element der Motivation und sogar eine Aufwertung ihrer Gemeinschaft bedeutet und nicht etwa eine Einmischung der Art Big Brother, wie die Beobachter aus dem Westen befürchteten.

Der Sprecher eines Dorfs bei Kankan (Guinea) liebte Brände. Statt sie zu bekämpfen, forderte er die Nachbardörfer auf, sie sollten dafür sorgen, dass sie sich ausbreiteten - obwohl diese Dörfer manchmal von diesen selbst gelegten Bränden zerstört wurden.

Als die Radioleute von den Gefahren der Wüstenbildung sprachen, meinte er: „Ich war soeben in Mekka. Da gibt es keine Bäume mehr, trotzdem sind die Leute nicht arm." Die Radiostation von Kankan gab diese Botschaft weiter, fügte aber eine Frage bei: „Sind Sie einverstanden mit diesem Dorfsprecher?“

Einige Tage später kam der Dorfsprecher und bat die Station, die Sendung abzusetzen. „Ich habe mich getäuscht“, räumte er kleinlaut ein. Das ganze Dorf hatte angefangen, nachzudenken, und sich gegen ihn gewandt.

Eine Evaluation der Kampagne zeigte die positiven Resultate der Kampagne. In den Gebieten, in denen die Sendung zu hören war, gingen die insgesamt durch solche Feuer verbrannten Flächen um 150'000 Hektaren zurück, was einer Abnahme um rund 40\% entspricht ${ }^{2}$. In ganz Westafrika nahmen die Frühfeuer zu, die Spätfeuer dagegen ab.

Die IKT arbeiten aber nicht immer zuverlässig. Die Verbreitung der Satellitenbilder, die jeden Monat verteilt werden sollten, verlief nicht reibungslos: Die elektronische Post funktioniert in den ländlichen Regionen unregelmässig, und Probleme mit dem Format und dem Internetzugang bremsen die Verbreitung und Nutzung der Bilder. Trotzdem wurden sie auf die eine oder andere Art fast überall hin weitergeleitet und von den Radiosendern kommentiert. Die Information funktioniert auch in die andere Richtung: Einige Dörfer bestätigten dem CSE, dass die per Satellit beobachteten Feuer wirklich stattfanden. Solche Informationen sind für die in Dakar stationierten Techniker sehr wertvoll.

\section{NOTES}

1. http://www.cse.sn.

2. Die klimatischen Bedingungen spielten sicher auch eine Rolle bei diesem spektakulären Resultat. 


\section{AUTHOR}

MARTIN FAYE

Direktor von Intermédia Afrique, Senegal. 